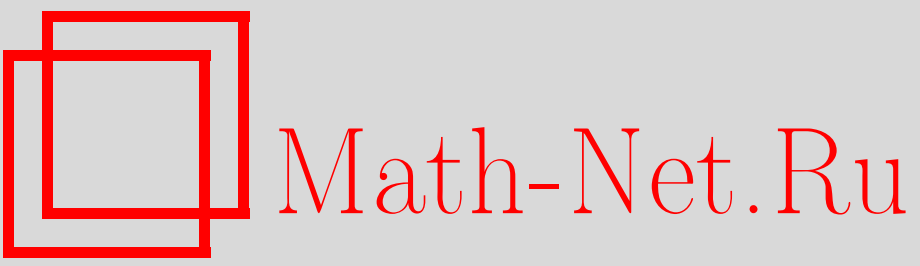

В. М. Бухштабер, Т. Е. Панов, Момент-угол комплексы и комбинаторика симплициальных многообразий, УМH, 2000, том 55, выпуск 3, 171-172

DOI: https://doi.org/10.4213/rm297

Использование Общероссийского математического портала Math-Net.Ru подразумевает, что вы прочитали и согласны с пользовательским соглашением

http://www.mathnet.ru/rus/agreement

Параметры загрузки:

IP: 54.172 .240 .79

26 апреля 2023 г., 15:32:37 


\title{
МОМЕНТ-УГОЛ КОМПЛЕКСЫ И КОМБИНАТОРИКА СИМПЛИЦИАЛЬНЫХ МНОГООБРАЗИЙ
}

\author{
В. М. БУХШТАБЕР, Т. Е. ПАНОВ
}

Пусть $\rho:\left(D^{2}\right)^{m} \rightarrow I^{m}$ - проекция на пространство орбит для диагонального действия тора $T^{m}$ на единичном полидиске $\left(D^{2}\right)^{m} \subset \mathbb{C}^{m}$. Каждая грань куба $I^{m}=[0,1]^{m}$, рассматриваемого как кубический комплекс, имеет вид

$$
F_{I \subset J}=\left\{\left(y_{1}, \ldots, y_{m}\right) \in I^{m}: y_{i}=0 \text { при } i \in I, y_{j}=1 \text { при } j \notin J\right\},
$$

где $I \subset J$ - два подмножества множества $[m]=\{1, \ldots, m\}$. Для каждой грани $F_{I \subset J}$ положим $B_{I \subset J}:=\rho^{-1}\left(F_{I \subset J}\right)$. Если \#I=i,\#J=j, то $B_{I \subset J} \cong\left(D^{2}\right)^{j-i} \times T^{m-j}$.

ОПредЕЛЕниЕ 1 . Пусть $C$-некоторый кубический подкомплекс в $I^{m}$. Момент-угол комnлексом $\mathrm{ma}(C)$ называется разбиение $T^{m}$-инвариантного подмножества $\rho^{-1}(C) \subset\left(D^{2}\right)^{m}$ на блоки $B_{I \subset J}$, соответствующие граням $F_{I \subset J}$ комплекса $C$.

Изучение эквивариантной топологии момент-угол комплексов позволяет решать задачи комбинаторики кубических разбиений. В настоящей работе мы демонстрируем возможности такого подхода в случае кубических разбиений, определяемых симплициальными комплексами. Пусть $K^{n-1}-(n-1)$-мерный симплициальный комплекс с $m$ вершинами и $|K|-$ соответствующий полиэдр. Если $I=\left\{i_{1}, \ldots, i_{k}\right\} \subset[m]$ является симплексом $K$, то мы будем писать $I \in K$. Введем два канонических кубических подкомплекса в $I^{m}$ :

$$
\operatorname{cub}(K)=\left\{F_{I \subset J}: J \in K, I \neq \varnothing\right\}, \quad \operatorname{cc}(K)=\left\{F_{I \subset J}: J \in K\right\} .
$$

Лемма 2. Как топологическое пространство, комплекс $\operatorname{cub}(K)$ гомеоморфен $|K|$, а $\operatorname{cc}(K)$ гомеоморфен конусу $|\operatorname{cone}(K)|$.

Кубический комплекс сс $(K)$ был введен в [1] и затем изучался в [2]. Кубический комплекс $\operatorname{cub}(K)$ появился в [3].

a)

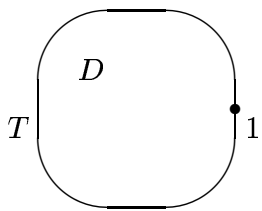

б)

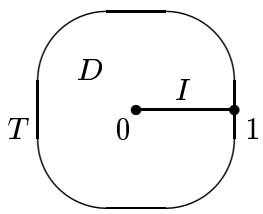

Обозначим момент-угол комплексы, соответствующие $\operatorname{cub}(K)$ и $\operatorname{cc}(K)$ через $\mathscr{W}_{K}$ и $\mathscr{Z}_{K}$ соответственно. Фиксируем клеточное разбиение полидиска $\left(D^{2}\right)^{m}$, при котором каждый $D^{2}$ представляется в виде объединения 0 -мерной клетки 1,1 -мерной клетки $T$ и 2 -мерной клетки $D$ (см. рис. а)). Каждая клетка в $\left(D^{2}\right)^{m}$ представляется в виде произведения клеток $D_{i}$, $T_{i}, 1_{i}, i=1, \ldots, m$, т.е. имеет вид $D_{I} T_{J} 1_{[m] \backslash I \cup J}$, где $I, J$ - попарно непересекающиеся подмножества $[m]$. Положим $D_{I} T_{J}:=D_{I} T_{J} 1_{[m] \backslash I \cup J}$. При этом $\mathscr{Z}_{K}$ является клеточным подкомплексом в $\left(D^{2}\right)^{m}$, состоящим из всех клеток $D_{I} T_{J}$, для которых $I \in K$.

Лемма 3. Вложение $T^{m}=\rho^{-1}(1, \ldots, 1) \hookrightarrow \mathscr{Z}_{K}$ является клеточным отображением, гомотопным отображсению в точку.

Как показано в [2], для произвольного поля $\mathbf{k}$ имеет место изоморфизм алгебр:

$$
H^{*}\left(\mathscr{Z}_{K}\right) \cong \operatorname{Tor}_{\mathbf{k}\left[v_{1}, \ldots, v_{m}\right]}(\mathbf{k}(K), \mathbf{k}) \cong H^{*}\left[\mathbf{k}(K) \otimes \Lambda\left[u_{1}, \ldots, u_{m}\right], d\right],
$$

где $\mathbf{k}(K)$ - кольо Стенли-Райснера комплекса $K$, а дифференциал $d$ определяется как $d\left(v_{i}\right)=0$, $d\left(u_{i}\right)=v_{i}, i=1, \ldots, m$. Tor-алгебра из (1) естественным образом является биградуированной, причем $\operatorname{bideg}\left(v_{i}\right)=(0,2), \operatorname{bideg}\left(u_{i}\right)=(-1,2)$. Вычисление кольца $H^{*}\left(\mathscr{Z}_{K}\right)$ позволило решить

Работа выполнена при поддержке Российского фонда фундаментальных исследований (грант № 99-01-00090). 
задачу описания мультипликативной структуры когомологий дополнения конфигурации координатных подпространств в $\mathbb{C}^{m}[2]$.

В [1] был введен подкомплекс $\mathscr{C}^{*}(K) \subset \mathbf{k}(K) \otimes \Lambda\left[u_{1}, \ldots, u_{m}\right]$, порожденный мономами $u_{J}$ и $v_{I} u_{J}$, для которых $I \cap J=\varnothing, I \in K$, и показано, что когомологии $\mathscr{C}^{*}(K)$ также совпадают с когомологиями $\mathscr{Z}_{K}$. Обозначим через $\mathscr{C}_{*}\left(\mathscr{Z}_{K}\right)$ и $\mathscr{C}^{*}\left(\mathscr{Z}_{K}\right)$ соответственно цепной и коцепной комплексы клеточного разбиения $\mathscr{Z}_{K}$ типа а).

Теорема 4. Пусть $\left(D_{I} T_{J}\right)^{*} \in \mathscr{C}^{*}\left(\mathscr{Z}_{K}\right)-$ кеточная коцепь, двойственная клетке $D_{I} T_{J} \in \mathscr{Z}_{K}$. Cоответствие $v_{I} u_{J} \mapsto\left(D_{I} T_{J}\right)^{*}$ устанавливает канонический изоморфизм комплексов $\mathscr{C}^{*}(K)$ и $\mathscr{C}^{*}\left(\mathscr{Z}_{K}\right)$, каждый из которых вычисляет $H^{*}\left(\mathscr{Z}_{K}\right)$.

Пара $\left(\mathscr{Z}_{K}, T^{m}\right)$ приобретает биградуированную клеточную структуру, где $\operatorname{bideg}\left(D_{i}\right)=$ $(0,2), \operatorname{bideg}\left(T_{i}\right)=(-1,2)$. Пусть $b_{-q, 2 p}\left(\mathscr{Z}_{K}, T^{m}\right)=\operatorname{dim} H_{-q, 2 p}\left[\mathscr{C}_{*}\left(\mathscr{Z}_{K}, T^{m}\right)\right]$. Фиксируем новое клеточное разбиение полидиска $\left(D^{2}\right)^{m}$, при котором каждый диск $D^{2}$ разбивается на 5 клеток $D, T, I, 1,0$ (см. рис. б)). Это позволяет ввести на $\mathscr{W}_{K}$ биградуированную клеточную структуру и определить числа $b_{q, 2 p}\left(\mathscr{W}_{K}\right)=\operatorname{dim} H_{q, 2 p}\left[\mathscr{C}_{*}\left(\mathscr{W}_{K}\right)\right]$. Положим

$$
\chi\left(\mathscr{Z}_{K}, T^{m} ; t\right)=\sum_{p, q}(-1)^{q} b_{-q, 2 p}\left(\mathscr{Z}_{K}, T^{m}\right) t^{2 p}, \quad \chi\left(\mathscr{W}_{K} ; t\right)=\sum_{p, q}(-1)^{q} b_{q, 2 p}\left(\mathscr{W}_{K}\right) t^{2 p} .
$$

Пусть $f_{i}$ - число $i$-симплексов $K$ и $\left(h_{0}, \ldots, h_{n}\right)$ - $h$-вектор, определяемый из соотношения $h_{0} t^{n}+\cdots+h_{n-1} t+h_{n}=(t-1)^{n}+f_{0}(t-1)^{n-1}+\cdots+f_{n-1}$.

Теорема 5. Положим $h(t)=h_{0}+h_{1} t+\cdots+h_{n} t^{n}$. Тогда

$$
\begin{aligned}
\chi\left(\mathscr{Z}_{K} ; T^{m}, t\right) & =\left(1-t^{2}\right)^{m-n} h\left(t^{2}\right)-\left(1-t^{2}\right)^{m}, \\
\chi\left(\mathscr{W}_{K} ; t\right) & =\left(1-t^{2}\right)^{m-n} h\left(t^{2}\right)+(-1)^{n-1} h_{n}\left(1-t^{2}\right)^{m} .
\end{aligned}
$$

Лемма 6. Если $|K| \cong S^{n-1}$, то $\mathscr{Z}_{K}$ является замкнутым многообразием.

Пусть теперь $K^{n-1}$ - симплициальное многообразие. Тогда комплекс $\mathscr{Z}_{K}$, вообще говоря, не является многообразием, но удаляя малую окрестность $U_{\varepsilon}\left(T^{m}\right)$ тора $T^{m}=\rho^{-1}(1, \ldots, 1)$, мы получаем многообразие $W_{K}=\mathscr{Z}_{K} \backslash U_{\varepsilon}\left(T^{m}\right)$ с краем $\partial W_{K}=|K| \times T^{m}$.

Теорема 7. Многообразие с краем $W_{K}$ әквивариантно гомотопически әквивалентно комплексу $\mathscr{W}_{K}$. Имеет место гомеоморфизм пар $\left(W_{K}, \partial W_{K}\right) \rightarrow\left(\mathscr{Z}_{K}, T^{m}\right)$.

Из относительной двойственности Пуанкаре для $W_{K}$ вытекает соотношение $\chi\left(\mathscr{W}_{K} ; t\right)=$ $(-1)^{m-n} t^{2 m} \chi\left(\mathscr{Z}_{K}, T^{m} ; \frac{1}{t}\right)$. Отсюда и из теоремы 5 получаем

СледСтвиЕ 8. Пусть $K^{n-1}-$ симплициальное многообразие. Тогда

(2) $\quad h_{n-i}-h_{i}=(-1)^{i}\left(h_{n}-1\right)\left(\begin{array}{c}n \\ i\end{array}\right)=(-1)^{i}\left(\chi\left(K^{n-1}\right)-\chi\left(S^{n-1}\right)\right)\left(\begin{array}{c}n \\ i\end{array}\right), \quad i=0,1, \ldots, n$.

Записав соотношения (2) в терминах $f$-вектора, мы приходим к более сложным соотношениям, полученным в [4], [5]. При $|K|=S^{n-1}$ следствие 8 дает классические соотношения Дена-Соммервилла. Для случая PL-многообразий топологическая инвариантность чисел $h_{n-i}-h_{i}$, вытекающая из следствия 8 , была установлена в [6; (7.11)].

Детальное изложение результатов см. в http://xxx.lanl.gov/abs/math.AT/0005199. Авторы выражают благодарность О.Р. Мусину который в ходе полезных обсуждений результатов работы обратил наше внимание на работы [4], [6].

\section{СПИСОК ЛИТЕРАТУРЫ}

[1] Бухштабер В. М., Панов Т. Е. // Труды МИРАН. 1999. Т. 225. С. 96-131. [2] Бухштабер В. М., Панов Т. Е. // Записки научных семинаров ПОМИ. 2000. Т. 266. С. 29-50. [3] Штанько М. А., Штогрин М. И. // УМН. 1992. Т. 47. №1. С. 219-220. [4] Chen В., Yan M. // Труды МИРАН. 1998. Т. 221. С. 305-319. [5] Klee V. // Canadian J. Math. 1964. V. 16. P. 517-531. [6] Pachner U. // European J. Combin. 1991. V. 12. P. 129-145.

Московский государственный университет им. М. В. Ломоносова

E-mail: buchstab@mech.math.msu.su; tpanov@mech.math.msu.su
Принято редколлегией 03.04.2000 